\title{
Recursive Receivers for Diversity Channels With Correlated Flat Fading
}

\author{
Van Khanh Nguyen, Langford B. White, Senior Member, IEEE, Emmanuel Jaffrot, Marcella Soamiadana, and \\ Inbar Fijalkow, Member, IEEE
}

\begin{abstract}
This paper addresses the design and performance of time-recursive receivers for diversity based communication systems with flat Rayleigh or Ricean fading. The paper introduces a general state-space model for such systems, where there is temporal correlation in the channel gain. Such an approach encompasses a wide range of diversity systems such as spatial diversity, frequency diversity, and code diversity systems which are used in practice. The paper describes a number of noncoherent receiver structures derived from both sequence and $a$ posteriori probabilitybased cost functions and compares their performance using an orthogonal frequency-division multiplex example. In this example, the paper shows how a standard physical delay-doppler scattering channel model can be approximated by the proposed state-space model. The simulations show that significant performance gains can be made by exploiting temporal, as well as diversity channel correlations. The paper argues that such time-recursive receivers offer some advantages over block processing schemes such as computational and memory requirement reductions and the easier incorporation of adaptivity in the receiver structures.
\end{abstract}

Index Terms-Diversity, fading channels, space-time coding, wireless communications.

\section{INTRODUCTION}

$\mathbf{I}$ N RECENT years, there has been an increasing emphasis on exploitation of different types of diversity in communications systems, particularly those which utilize a wireless transmission medium. Examples of types of diversity which have been exploited to improve the performance of digital communications systems are time diversity (also known as fractional sampling) [1], spatial diversity via the use of several transmitter, and/or receiver antennae [2], [3], code diversity (as used in spread-spectrum communications), and frequency diversity such as used in orthogonal frequency-division multiplexing (OFDM) systems. Also, these different types of diversity can also be combined, for example, a multicarrier

Manuscript received May 1, 2002; revised October 30, 2002. The work of V. K. Nguyen and L. B. White was done while visiting ETIS. The work of L. B. White was supported in part by ETIS on a French RNRT (National Telecommunications Research Network) Project. This paper was presented in part at the Australian Communication Theory Workshop, Canberra, February 2002 and at the EUSIPCO, Toulouse, September 2002.

V. K. Nguyen and L. B. White are with the Centre for Internet Technology Research, Adelaide University, Adelaide, South Australia 5005, Australia (e-mail: vknguyen@eleceng.adelaide.edu.au; 1white@eleceng.adelaide.edu.au).

E. Jaffrot is with the Ecole Nationale Superieure des Techniques Avancees (ENSTA), Paris, France (e-mail: jaffrot@ensta.fr).

M. Soamiadana and I. Fijalow are with the ETIS and also with Ecole Nationale Supérieure de l'Electronique et ses Applications (ENSEA), University of Cergy-Pontoise-CNRS, 95014 Cergy-Pontoise Cedex, France (e-mail: soamiada@ensea.fr; fijalkow@ensea.fr).

Digital Object Identifier 10.1109/JSAC.2003.810332 code-division multiple-access (CDMA) system. In these types of systems, the presence of a number of different diversity channels can be used to improve the system performance by transmitting and/or receiving the same information symbols simultaneously across a number of channels. This goal can be achieved by introducing coding in both the temporal and diversity dimensions. This paper is concerned with the design of receivers for such diversity systems.

Given that there are many different types of diversity which may be exploited, it is desirable to develop a generalized and unified taxonomy for system modeling and signal processing for such systems. This framework can be used to derive various types of receiver structures which are applicable to any type of diversity system. In particular, we will consider systems where the channel is modeled by vector time series where each element of the vector represents the complex gain of a different diversity channel. We will assume that these gains are correlated in both the diversity dimension (i.e., the gains of different channels are statistically dependent at each time), and the temporal direction. Temporal correlation can arise from doppler effects [4] and from oversampling as examples. We particularly focus on state-space channel models which lead naturally to time-recursive receiver structures. These structures generally tend to offer reduced computational complexity and memory requirements when compared with block processing systems. Another potential advantage of time-recursive receivers is the ease of development of adaptive processing algorithms, however, we do not address this issue in this paper.

Since the computational complexity of the optimal receiver is generally too large to be feasible, the paper focuses on the derivation and comparison of a number of suboptimal receivers which have computational complexity which is linear in the size of the transmission codebook. In this paper, we will tend to focus on single-user systems where the general aim of the receiver is to exploit the temporal and diversity correlation to improve performance. Much of what is considered here also applies to the multiuser case, where the diversity correlation leads to undesirable interference between users. The task of the receiver is then to mitigate this interference.

In this paper, we address only flat-fading channels. Related work which addresses, in a different way, the frequency selective space-time case, can be found in [5]. Recent work in the space-time coding context [6] utilizes fading models, where correlation between the diversity channels is included. Of course, such correlation is an inherent feature in CDMA systems, and the main focus of various multiuser detection schemes (see [7] and [8] for an overview) is the mitigation of the detrimental effects of such correlation. However, there 
has been significantly less published concerning the presence of, and exploitation of temporal correlation in the channel. We believe that this work makes the following contributions: 1) a generalized state-space model for diversity communication systems; 2) development of a number of time-recursive receiver structures which exploit temporal correlations in the channel; and 3) a performance comparison of these proposed receivers. In addition, we examine the issue of matching our proposed model to a physical correlation model for delay-doppler channels [9], [10]. Some of the proposed receivers are well-known, but others are novel, especially in the context of their application in this problem.

There has been considerable interest also in the code design issue for diversity systems, particularly space-time diversity systems (see, e.g., [6] and [11]-[14]). We do not directly address the design issue in this paper, but we will make some pertinent observations regarding code design in the context of the OFDM example presented in Section IV.

The layout of the paper is as follows. In Section II, we introduce our model of a general diversity system in complex number form, and then in a real quadrature signal form. The latter will form the basis for the receiver design. The model for the received signal constitutes the measurement equation for a time varying state-space system. Our model for the diversity path gains is cast as a quadrature Gauss-Markov process which constitutes the state equation for the state-space system. We also give some examples, which are by no means exhaustive, of how some well-known examples of diversity systems may be cast within our framework. In Section III, we introduce a number of candidate noncoherent receiver structures which are based on the state-space diversity model from Section II. In Section IV, we detail a specific application of our techniques to the OFDM system. The diversity dimension here is frequency. We examine a general physical delay-doppler scattering model for path correlations as a function of time and frequency, and detail a technique which permits approximation of this correlation function by the signal model introduced in Section II. Some issues relating to partial diversity and an associated model reduction method are discussed. Finally, we present some simulation results for the OFDM case, which compare the performance of our candidate receivers to the coherent case (path gains known by the receiver), and to standard approaches which do not exploit the time correlation.

\section{COMMUNICATION SYSTEM MODEL}

In this paper, we consider a communication system consisting of $L \geq 1$ diversity paths. These diversity paths are assumed to undergo Rayleigh/Ricean flat fading. The path gains are correlated with each other and are also temporally correlated. At each time $t$, we map $p$ bits of the input data stream onto one of the codewords of the set $\tilde{\chi}=\left\{\tilde{X}^{(1)}, \tilde{X}^{(2)}, \ldots, \tilde{X}^{(K)}\right\}$, where $\tilde{X}^{(k)} \in \mathbb{C}^{M \times L}$ and $K=2^{p}$. The codeword is then transmitted serially across the $L$ channels, i.e., column $\ell$ of the selected code is transmitted serially across diversity path $\ell$. We assume that the receiver measures a linear superposition of the $L$ diversity paths. Thus, the received signal in block of $M$ complex samples ( $M$ is the temporal dimension) can be written as

$$
\tilde{y}_{t}=\tilde{X}_{t} \tilde{h}_{t}+\tilde{n}_{t}
$$

where $\tilde{h}_{t} \in \mathbb{C}^{L}$ is the channel gains and $\tilde{n}_{t} \in \mathbb{C}^{M}$ is a zero-mean complex circular white Gaussian noise sequence with covariance $2 \sigma^{2} I_{M}$. We now give several examples of how this model may be applied to represent different diversity systems.

\section{A. Examples}

1) Space-time coded system with a single receive antenna. Here, we have $L$ transmitters and the diversity is obtained by virtue of the $L$ paths from the transmitters to the single receiver. In each block of $M$ time samples, transmitter $\ell$ transmits the sequence $\left[\tilde{X}_{t}\right]_{m, \ell}$ for $m=0, \ldots, M-1$. The diversity gains $\tilde{h}_{t}$ represent the complex flat fading gains on each spatial path. The codes $\tilde{X}^{(k)}$ are known as space-time codes [6].

2) Space-time coded system with $N$ receive antennae. Let the channel gain between transmitter $\ell$ and receiver $n$ be given by $\left[\tilde{h}_{t}\right]_{n L+\ell}$ for $\ell=0, \ldots, L-1$ and $n=0, \ldots, N-1$. Let $\left[\tilde{y}_{t}\right]_{n M+m}$ denote the received signal for sample $m$ (of block $t$ ) and receiver $n$, for $m=0, \ldots, M-1$ and $n=0, \ldots, N-1$, and $\left[\tilde{n}_{t}\right]_{n M+m}$ for the corresponding noise sample. Then, (1) holds with the appropriate dimension changes, and the $M N \times L N$ code matrix is formed by making a block diagonal matrix with $N \times N$ blocks (each of size $M \times L$ ) with the same space-time code on each diagonal block and zeros elsewhere.

3) OFDM. Here, the diversity dimension is frequency, so we can regard OFDM as a frequency-time coded system. Suppose we seek to transmit $n$ complex symbols in a vector $s_{t}$ for time period $t$. We use a precoder matrix $C \in \mathbb{C}^{L \times n}$ to map the $n$ symbols onto the $L$ orthogonal frequency channels. Here, $M=L$. The signal transmitted on channel $\ell$ will be the $\ell$ th element of the vector $C s_{t}$. The cyclic prefix which is added at the transmitter and stripped off at the receiver turns the time and frequency-selective fading into a time fading on each carrier, see [9]. Thus, the received signal for symbol period $t$ is the superposition

$$
\left[\bar{y}_{t}\right]_{m}=\sum_{\ell=0}^{L-1}\left[\tilde{h}_{t}\right]_{\ell}\left[C s_{t}\right]_{\ell} e^{2 \pi i m \ell / L}+\left[\tilde{n}_{t}\right]_{m}
$$

where $\left[\tilde{h}_{t}\right]_{\ell}$ denotes the complex gain of channel $\ell$, and $\tilde{n}_{t}$ is additive Gaussian white noise. Thus, we have the form of (1) where the code matrix has the form $\tilde{X}_{t}=F^{H} S_{t}$ where $S_{t}$ is a $L \times L$ diagonal matrix with its diagonal elements being the entries of the vector $C s_{t}$, and $F$ is the Fourier matrix $[F]_{m l}=e^{-2 \pi i m \ell / L}$. More details on OFDM as a diversity system are given in Section IV.

4) Code-division multiple-access (CDMA) uplink. This is an example of a multiuser diversity system. In this case, column $\ell$ of the code matrix is the spreading code for user $\ell$ multiplied by the complex modulation symbol for user $\ell$ at symbol time $t$. Thus, if $W \in \mathbb{C}^{M \times L}$ denotes a matrix of the length $M$ spreading codes for active users, and $S_{t}$ is a diagonal matrix of size $L \times L$ with the diagonal elements being the modulation symbols of the active users 
for symbol period $t$, then the associated diversity code is $\tilde{X}_{t}=W S_{t}$. The path gains in this case are the propagation gains from each user mobile station to the base station. In this case, our receiver designs will constitute noncoherent multiuser detectors.

\section{B. Real Quadrature Model Form}

It will be convenient for the resulting receiver derivations, to utilize a real quadrature model for the signal. Let denote the real and imaginary part of an arbitrary complex matrix (or vector) $\tilde{\theta}_{t}$ by $\theta_{t}^{r}$ and $\theta_{t}^{i}$, respectively. The complex received signal (1) can be modeled into quadrature form by

$$
y_{t}=\left[\begin{array}{c}
y_{t}^{r} \\
y_{t}^{i}
\end{array}\right]=X_{t} h_{t}+n_{t}
$$

where $y_{t} \in \mathbb{R}^{2 M}, h_{t} \in \mathbb{R}^{2 L}$ and $X_{t} \in \mathbb{R}^{2 M \times 2 L}$ are given by

$$
h_{t}=\left[\begin{array}{c}
h_{t}^{r} \\
h_{t}^{i}
\end{array}\right], \quad n_{t}=\left[\begin{array}{c}
n_{t}^{r} \\
n_{t}^{i}
\end{array}\right], \quad X_{t}=\left[\begin{array}{cc}
X_{t}^{r} & -X_{t}^{i} \\
X_{t}^{i} & X_{t}^{r}
\end{array}\right] .
$$

The transmitted codeword $X_{t}$ is now selected from the corresponding set $\chi=\left\{X^{(1)}, X^{(2)}, \ldots, X^{(K)}\right\}$. Here, $n_{t} \in \mathbb{R}^{2 M}$ is a zero-mean Gaussian white noise vector with covariance $\sigma^{2} I_{2 M}$

\section{Time-Correlated Rayleigh/Ricean Flat-Fading Channel Model}

In this section, we define the model for the diversity path gains $\tilde{h}_{t}$ in both complex and real quadrature form. The channel gains are modeled as a first-order vector Gauss-Markov process expressed in complex form by

$$
\tilde{h}_{t+1}=\tilde{A} \tilde{h}_{t}+\tilde{v}_{t}
$$

where $\tilde{A} \in \mathbb{C}^{L \times L}$ is a known, stable matrix and $\tilde{v}_{t}$ is a complex Gaussian white noise process with mean $\tilde{\mu}$ and covariance $\tilde{Q}$. We assume that at the initial time $t=0$ that $\tilde{h}_{0}$ is chosen to be Gaussian with the steady state statistics

$$
E\left\{\tilde{h}_{0}\right\}=(I-\tilde{A})^{-1} \tilde{\mu}
$$

and $\operatorname{Cov}\left\{\tilde{h}_{0}\right\}=\tilde{P}$ where $\tilde{P}$ satisfies the Lyapunov equation $\tilde{A} \tilde{P} \tilde{A}^{H}+\tilde{Q}=\tilde{P}$. Thus, $\tilde{h}_{t}$ will be a stationary process with mean given by (6) and with autocorrelation matrix

$$
E\left\{\tilde{h}_{t} \tilde{h}_{t-\tau}^{H}\right\}= \begin{cases}\tilde{A}^{\tau} \tilde{P}, & \tau \geq 0 \\ \tilde{P}\left(\tilde{A}^{H}\right)^{-\tau}, & \tau<0 .\end{cases}
$$

This channel model is general and permits both temporal and diversity correlations in the gains. Several channel models considered in [6], [12], and [13] for the space-time coded system can be considered as special cases of this model. The quasi-static independent and identical distributed (i.i.d.) fading model used in [12] can be represented by this general model with $\tilde{A}=0$ and $\tilde{P}=I$, where 0 is the matrix of all zeros. Setting $\tilde{A}=0$ and $\tilde{P}=E\left\{\tilde{h}_{t} \tilde{h}_{t}^{H}\right\} \neq I$, this model corresponds to the quasi-static correlated fading channel model in [6]. The time-varying i.i.d fading channel model in [16] can be cast into this general model by having $\tilde{P}=I$ and $\tilde{A}=\alpha I$ where $\alpha<1$.
The analogous real quadrature form for (5) is

$$
h_{t+1}=A h_{t}+v_{t}
$$

where

$$
A=\left[\begin{array}{cc}
A^{r} & -A^{i} \\
A^{i} & A^{r}
\end{array}\right], \quad v_{t}=\left[\begin{array}{c}
v_{t}^{r} \\
v_{t}^{i}
\end{array}\right]
$$

The process $v_{t} \in \mathbb{R}^{2 L}$ is a Gaussian white noise process with mean $\mu$ and covariance matrix $Q$ given by

$$
\mu=\left[\begin{array}{l}
\mu^{r} \\
\mu^{i}
\end{array}\right], \quad Q=\left[\begin{array}{ll}
Q_{r r} & Q_{r i} \\
Q_{r i}^{T} & Q_{i i}
\end{array}\right]
$$

with $Q_{r r}+Q_{i i}=Q^{r}$ and $Q_{r i}^{T}-Q_{r i}=Q^{i}$. The mean and autocorrelation sequence for $h_{t}$ are then given by

$$
\begin{array}{r}
\nu=E\left\{h_{t}\right\}=(I-A)^{-1} \mu \\
E\left\{h_{t} h_{t-\tau}^{T}\right\}= \begin{cases}A^{\tau} P & \tau \geq 0 \\
P\left(A^{T}\right)^{-\tau} & \tau<0\end{cases}
\end{array}
$$

where

$$
P=\left[\begin{array}{ll}
P_{r r} & P_{r i} \\
P_{r i}^{T} & P_{i i}
\end{array}\right]
$$

with $P_{r r}+P_{i i}=P^{r}$ and $P_{r i}^{T}-P_{r i}=P^{i}$. Equation (3) and (8) are, respectively, the observation and state equations for a state-space model of our received signal. The mean and autocorrelations for $h_{t}$ can be shown to be identical to the analogous means and autocorrelations of the complex sequence $\tilde{h}_{t}$. Thus, the magnitude gain of the channels is Ricean (if $\mu \neq 0$ ) or Rayleigh (if $\mu=0$ ) with joint correlations in the diversity and time dimensions given by (11). Equation (11) shows that the autocorrelation sequence has the form of a mixture of decaying complex exponentials (determined by the eigenvalues of the stable matrix $A$ ).

\section{RECEIVERS}

In this section, we describe a number of noncoherent receiver structures for estimating the code sequence $X_{t}$ in the absence of knowledge of the channel gains. The first class of receivers, which includes the generalized-likelihood ratio test (GLRT) [6], [14], [15], noncoherent maximum-likelihood (NCML) [6], [12], and maximum a posteriori probability (MAP) receivers, are well-known and do not exploit the temporal correlation in the signal. The NCML and MAP receivers, however, do exploit the correlation in the diversity dimension. The second class are Kalman filter-based receivers which includes the per-survivor processing (PSP), M-algorithm, a posteriori probability (APP), and iterative expected-maximization (EM) receivers. These receivers are based on the state-space model from Section II. The PSP, APP, M-algorithm, and EM algorithms are all well-known. However, the novelty of these receivers is that we incorporate Kalman filtering into these standard algorithms to exploit the temporal correlation in the signals and track the channel variations.

\section{A. Generalized-Likelihood Ratio Test (GLRT) Receiver}

When the probability distribution of the diversity path gains is unknown, a GLRT receiver can be used. The GLRT receiver 
computes the joint maximum likelihood estimate of the channel and the transmitted codeword. Thus, the transmitted codeword estimated by the GLRT receiver is

$$
\hat{X}_{t}^{\mathrm{GLRT}}=\arg \max _{X \in \chi} \sup _{h_{t}} p\left(y_{t} \mid X_{t}=X, h_{t}\right)
$$

where $p\left(y_{t} \mid X_{t}, h_{t}\right)$ is the data likelihood function, which is a Gaussian with mean $X_{t} h_{t}$ and covariance $\sigma^{2} I$. This is equivalent to

$$
\hat{X}_{t}^{\mathrm{GLRT}}=\arg \min _{k}\left\|P_{k}^{\perp} y_{t}\right\|^{2}
$$

where $P_{k}^{\perp}=I-X^{(k)}\left(X^{(k)^{T}} X^{(k)}\right)^{-1}\left(X^{(k)}\right)^{T}$ is the orthogonal projector to the codeword $X^{(k)}$.

\section{B. Noncoherent Maximum-Likelihood (NCML) Receiver}

In the situation where the probability distribution of the fading channel is known, one can use the NCML receiver. The NCML receiver maximizes the marginal density $p\left(y_{t} \mid X_{t}\right)$ over $X_{t}$

$$
\begin{aligned}
\hat{X}_{t}^{\mathrm{NCML}} & =\arg \max _{X \in \chi} p\left(y_{t} \mid X_{t}=X\right) \\
& =\arg \max _{X \in \chi} E_{h_{t}}\left\{p\left(y_{t}, h_{t} \mid X_{t}=X\right)\right\}
\end{aligned}
$$

where the expectation is with respect to the channel $h_{t}$. This is equivalent to choosing the codeword which minimizes the following cost function:

$$
J_{t}^{\mathrm{NCML}}(X)=\epsilon_{t}^{T}(X) \Omega_{t}^{-1}(X) \epsilon_{t}(X)+\log \operatorname{det} \Omega_{t}(X)
$$

where $\epsilon_{t}(X)=y_{t}-X \nu ; \Omega_{t}(X)=X P X^{T}+\sigma^{2} I ; \nu$ and $P$ are the mean and covariance of $h_{t}$. This receiver does not exploit the temporal correlation as it operates independently from codeword period to codeword period. However, it can be extended to account for the temporal correlation by incorporating multiple codewords into the decision metric (16), albeit at the cost of exponential increase in processing complexity.

\section{Maximum a Posteriori Probability (MAP) Receiver}

Another receiver which can be used when the statistics of the fading channel is known is the MAP receiver. The MAP receiver works by maximizing over $h_{t}$ and $X_{t}$, the APP $p\left(X_{t}, h_{t} \mid y_{t}\right) \propto p\left(y_{t} \mid h_{t}, X_{t}\right) p\left(h_{t}\right)$, assuming the codes are chosen from the codebook with uniform probability. That is

$$
\hat{X}_{t}^{\mathrm{MAP}}=\arg \max _{X \in \chi} \sup _{h_{t}} p\left(y_{t}, h_{t} \mid X_{t}=X\right)
$$

where

$$
p\left(y_{t} \mid X, h_{t}\right) p\left(h_{t}\right)=\mathcal{N}\left(y_{t}-X h_{t} ; \sigma^{2} I\right) \mathcal{N}\left(h_{t}-\nu ; P\right) .
$$

Here, $\mathcal{N}(x, P)$ denotes the Gaussian density

$$
\mathcal{N}(x, P)=\frac{1}{(2 \pi)^{N / 2}(\operatorname{det} P)^{1 / 2}} e^{-x^{T} P^{-1} x / 2}
$$

where $N$ is the dimension of the vector $x$. It can be shown that $p\left(y_{t} \mid X, h_{t}\right) p\left(h_{t}\right)$ can be expressed as

$$
\begin{aligned}
& p\left(y_{t} \mid X, h_{t}\right) p\left(h_{t}\right) \\
& \quad=\mathcal{N}\left(\epsilon_{t}(X) ; \Omega_{t}(X)\right) \mathcal{N}\left(h_{t}-\hat{h}_{t}(X) ; \hat{P}_{t}(X)\right)
\end{aligned}
$$

where

$$
\begin{aligned}
& \hat{h}_{t}(X)=\nu+P X^{T}\left(X P X^{T}+\sigma^{2} I\right)^{-1}\left(y_{t}-X \nu\right) \\
& \hat{P}_{t}(X)=P-P X^{T}\left(X P X^{T}+\sigma^{2} I\right)^{-1} X P .
\end{aligned}
$$

Clearly, $\hat{h}_{t}(X)$ maximizes $p\left(y_{t} \mid X, h_{t}\right) p\left(h_{t}\right)$ over $h_{t}$ and the maximized value is

$$
\begin{aligned}
p\left(y_{t} \mid X, \hat{h_{t}}(X)\right) p & \left(\hat{h_{t}}(X)\right) \\
& =\mathcal{N}\left(\epsilon_{t}(X) ; \Omega_{t}(X)\right) \mathcal{N}\left(0 ; \hat{P}_{t}(X)\right) .
\end{aligned}
$$

Thus, the cost function that the MAP receiver needs to minimize is the same as that of the noncoherent ML receiver plus an extra term $\log \operatorname{det} \hat{P}_{t}$ (neglecting constant terms)

$$
J_{t}^{\mathrm{MAP}}(X)=J_{t}^{\mathrm{NCML}}(X)+\log \operatorname{det} \hat{P}_{t}(X) .
$$

More detailed description of the GLRT, NCML, and MAP approaches can be found in [17].

The receivers described above do not exploit the temporal correlation of the fading channels and operate on each symbol period independently. In the following sections, we describe several receivers which take this temporal correlation into account when performing the detection.

\section{Sequence Estimation Approaches}

Consider a sequence of codewords $\left\{X_{0}, X_{1}, \ldots, X_{T-1}\right\}$ being transmitted during $T$ codeword intervals. In order to obtain an optimal solution which maximizes the likelihood $p\left(y_{0}, \ldots, y_{T-1} \mid X_{0}, \ldots, X_{T-1}\right)$, there must be a Kalman filter for each of the possible sequence (model). This direct implementation of the optimal receiver has a complexity of $O\left(K^{T}\right)$ which grows exponentially with the length of the sequence. Thus, even for small $T$, it may not be practical to use this method. Therefore, we need to examine other suboptimal methods. In this section, we describe two receivers which approximately optimize the likelihood of the transmitted sequence, i.e., $p\left(y_{0}, \ldots, y_{T-1} \mid X_{0}, \ldots, X_{T-1}\right)$.

1) Per-Survivor Processing (PSP) Receiver: In this section, we describe a receiver which approximates the optimal receiver by using the PSP method [18]. This method can be implemented via the Viterbi algorithm. In this method, there will be $K$ Kalman filters (KFs), each tuned to one of the $K$ codewords. Fig. 1 illustrates the structure of this receiver.

At time $t$, for each codeword the receiver calculates the loglikelihood from the possible paths. After computing the path metric, it retains the path with the largest log-likelihood and computes the channel estimate for this path. The approximately 


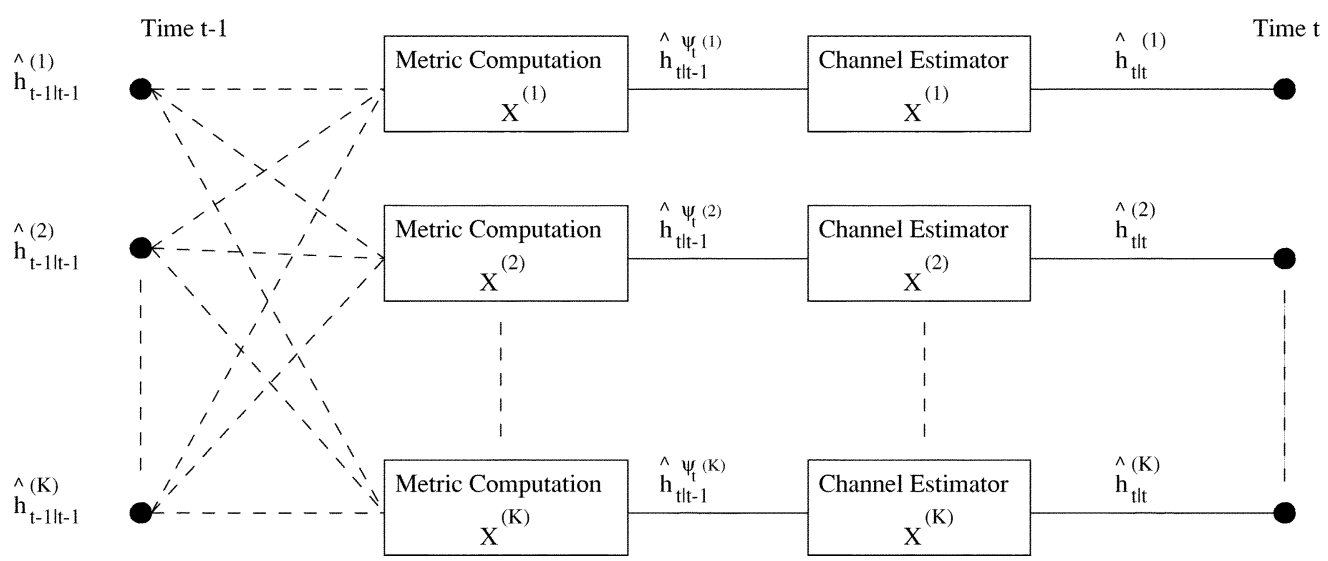

Fig. 1. Structure of PSP receiver.

optimal log-likelihood at time $t$ of the $k$ th Kalman filter tuned to $X_{t}=X^{(k)}$ can be evaluated according to

$$
\begin{aligned}
\delta_{t}^{(k)}= & \max _{j=1, \ldots, K} \\
& \cdot\left(\delta_{t-1}^{(j)}-\epsilon_{t}^{(j, k)^{T}} \Omega_{t}^{(j, k)^{-1}} \epsilon_{t}^{(j, k)}-\log \operatorname{det} \Omega_{t}^{(j, k)}\right)
\end{aligned}
$$

where $\epsilon_{t}^{(j, k)}$ is the innovation sequence of the path $X_{t-1}=X^{(j)}$ and $X_{t}=X^{(k)}$, and $\Omega_{t}^{(j, k)}$ is its associated covariance

$$
\begin{aligned}
\epsilon_{t}^{(j, k)} & =y_{t}-X^{(k)} \hat{h}_{t \mid t-1}^{(j)} \\
\Omega_{t}^{(j, k)} & =X^{(k)} \Sigma_{t \mid t-1}^{(j)} X^{(k)^{T}}+\sigma^{2} I .
\end{aligned}
$$

The predicted channel estimate $\hat{h}_{t \mid t-1}^{(j)}$ and its covariance $\Sigma_{t \mid t-1}^{(j)}$ of the path from $X_{t-1}=X^{(j)}$ are determined from the filtered channel estimate $\hat{h}_{t-1 \mid t-1}^{(j)}$ and its covariance $\Sigma_{t-1 \mid t-1}^{(j)}$ via the Kalman filter time update equations

$$
\begin{aligned}
& \hat{h}_{t \mid t-1}^{(j)}=A \hat{h}_{t-1 \mid t-1}^{(j)}+\mu \\
& \Sigma_{t \mid t-1}^{(j)}=A \Sigma_{t-1 \mid t-1}^{(j)} A^{T}+Q .
\end{aligned}
$$

Once the survivor path at $X_{t}=X^{(k)}$ is determined, the Kalman filter then estimates the channel associated with this path using the received signal at time $t$. Let the predecessor of $X_{t}=X^{(k)}$ which maximizes the log-likelihood $\delta_{t}^{(k)}$ be denoted by $\psi_{t}(k)$. The filtered channel estimate of the survivor path at time $t$ ending with $X_{t}=X^{(k)}$ can be evaluated by the Kalman filter measurement update equations

$$
\begin{aligned}
\hat{h}_{t \mid t}^{(k)} & =\hat{h}_{t \mid t-1}^{\left(\psi_{t}(k)\right)}+G_{t}^{\left(\psi_{t}(k)\right)}\left(y_{t}-X^{(k)} \hat{h}_{t \mid t-1}^{\left(\psi_{t}(k)\right)}\right) \\
G_{t}^{\left(\psi_{t}(k)\right)} & =\Sigma_{t \mid t-1}^{\left(\psi_{t}(k)\right)} X^{(k)^{T}}\left(X^{(k)} \Sigma_{t \mid t-1}^{\left(\psi_{t}(k)\right)} X^{(k)^{T}}+\sigma^{2} I\right)^{-1} \\
\Sigma_{t \mid t}^{(k)} & =\Sigma_{t \mid t-1}^{\left(\psi_{t}(k)\right)}-G_{t}^{\left(\psi_{t}(k)\right)} X^{(k)} \Sigma_{t \mid t-1}^{\left(\psi_{t}(k)\right)} .
\end{aligned}
$$

This method has a complexity of $O(K)$ which is a huge reduction as compare with the optimal detector. It is possible to develop an $O\left(K^{2}\right)$ version of this approach, in which each Kalman filter which tunes to $X_{t}$, produces $K$ filtered channel estimates, one for each survivor paths from time $t-1$. Once the channel estimates are made, it computes the path metric and selects the one which maximizes the log-likelihood. The computation of the path metric is the same as in (24), with the predicted channel estimate and its covariance replaced by the filtered channel estimates and its associated covariance.

2) M-Algorithm Receiver: The PSP method offers a much lower complexity than the optimal receiver. However, there are situations in which the codebook size, $K$, is too large which may prevent the PSP method from being used. Also the PSP receiver retains only one survivor path for each codeword at time $t$. This could be a disadvantage as the discarded paths at some codewords could have a much larger likelihood than the survivor paths at other codewords. Motivated by this observation, we developed a receiver which is based on the M-algorithm [19] for detecting the transmitted codeword. The complexity of this receiver can vary from $O(1)$ to $O(K)$ depending on the performance that we wish to obtain.

The M-algorithm receiver is implemented using a bank of $S$ Kalman filters, where $1 \leq S \leq K$. A pictorial description of the M-algorithm receiver is depicted in Fig. 2. This receiver works as follows: At the end of each symbol period, the receiver retains $S$ channel estimates with the largest log-likelihood. Suppose at the end of time $t-1$, the $S$ survivor channel estimates are $\hat{h}_{t-1 \mid t-1}^{(m)}$ for $m=1, \ldots, S$. At time $t$, for each codeword $X_{t}$, the receiver computes $S$ path metrics using the predicted channel estimates $\hat{h}_{t \mid t-1}^{(m)}=A \hat{h}_{t-1 \mid t-1}^{(m)}+\mu$. The log-likelihood of the path from the $m$ th survivor at time $t-1$ and $X_{t}=X^{(k)}$ is calculated by

$$
\tilde{\delta}_{t}^{(m, k)}=\delta_{t-1}^{(m)}-\epsilon_{t}^{(m, k)^{T}} \Omega_{t}^{(m, k)^{-1}} \epsilon_{t}^{(m, k)}-\log \operatorname{det} \Omega_{t}^{(m, k)}
$$

where $\delta_{t-1}^{(m)}$ is the log-likelihood of the survivor path $m$ at time $t-1, \epsilon_{t}^{(m, k)}=y_{t}-X^{(k)} \hat{h}_{t \mid t-1}^{(m)}$ and $\Omega_{t}^{(m, k)}=X^{(k)} \Sigma_{t \mid t-1}^{(m)} X^{(k)^{T}}+\sigma^{2} I$ are the innovation sequence and its covariance, respectively. Thus, there are $S K$ candidate paths in total and the M-algorithm selects $S$ paths with the largest log-likelihood. Let the path from the $m$ th survivor at time $t-1$ and $X_{t}=X^{(k)}$ being selected by the M-algorithm as the sth survivor path at time $t$. The filtered 


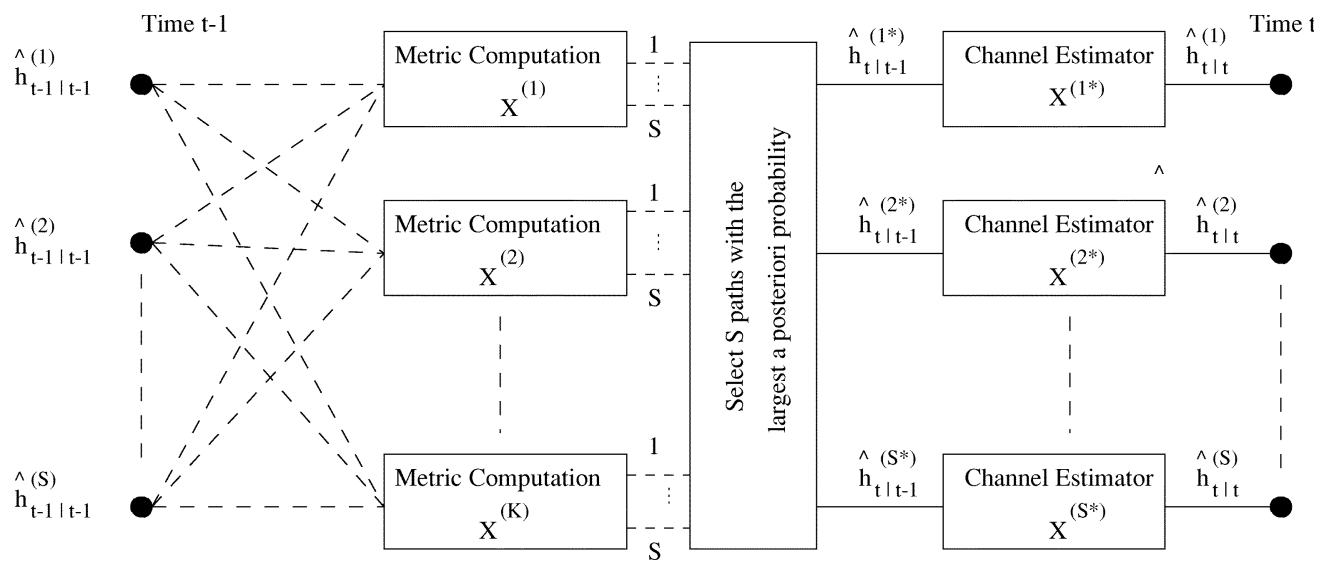

Fig. 2. Structure of M-algorithm receiver.

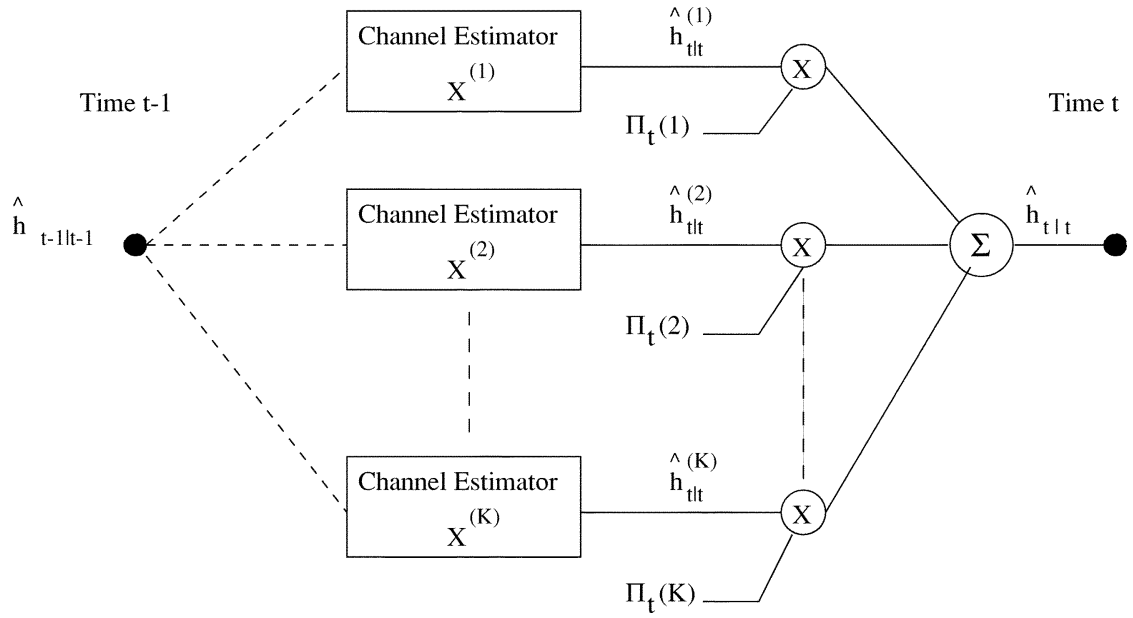

Fig. 3. Structure of APP receiver.

channel estimate of this sth survivor path can then be updated by using a Kalman filter tuned to $X_{t}=X^{(k)}$. Thus

$$
\begin{aligned}
\hat{h}_{t \mid t}^{(s)} & =\hat{h}_{t \mid t-1}^{(m)}+G_{t}^{(m, k)}\left(y_{t}-X^{(k)} h_{t \mid t-1}^{(m)}\right) \\
G_{t}^{(m, k)} & =\Sigma_{t \mid t-1}^{(m)} X^{(k)^{T}}\left(X^{(k)} \Sigma_{t \mid t-1}^{(m)} X^{(k)^{T}}+\sigma^{2} I\right)^{-1} \\
\Sigma_{t \mid t}^{(s)} & =\Sigma_{t \mid t-1}^{(m)}-G_{t}^{(m, k)} X^{(k)} \Sigma_{t \mid t-1}^{(m)} .
\end{aligned}
$$

In both the PSP and M-algorithm, the optimal sequence is extracted by backtracking through the survivor paths. On-line versions can also be derived.

\section{E. Codeword by Codeword Estimation}

The receivers described previously are used for sequence estimation. In this section, we describe two other techniques which detect the transmitted codeword at each codeword interval. Thus, these receivers can be used in applications which cannot tolerate the delays associated with the Viterbi algorithm backtracking, or when we desire to incorporate adaptivity to the channel statistics.

1) A Posteriori Probability (APP) Receiver: Unlike the receivers described previously which retain several estimates of the fading channels at the end of each codeword interval, this re- ceiver produces a single channel estimate which is the weighted sum of all the estimates. This receiver is implemented by using a bank of $K$ Kalman filters, where each Kalman filter is tuned to one of the codewords. Fig. 3 illustrates the structure of this receiver.

Based on the assumed signal model, each Kalman filter gives a conditional channel estimate. These channel estimates will then be weighted and summed to yield the mean channel estimate. The weighting coefficient of the channel estimate $\hat{h}_{t \mid t}^{(k)}$ is the APP that $X_{t}=X^{(k)}$, i.e., $p\left(X_{t}=X^{(k)} \mid y_{0}, \ldots, y_{t}\right)$. Using the Bayes rule, the APP that $X_{t}$ take on each value in $\chi$ is computed according to

$$
\begin{aligned}
\Pi_{t}(k) & =p\left(X_{t}=X^{(k)} \mid y_{0}, \ldots, y_{t}\right) \\
& =c_{t} p\left(y_{t} \mid y_{0}, \ldots, y_{t-1}, X_{t}=X^{(k)}\right)
\end{aligned}
$$

where

$$
p\left(y_{t} \mid y_{0}, \ldots, y_{t-1}, X_{t}=X^{(k)}\right)=\mathcal{N}\left(\epsilon_{t}^{(k)} ; \Omega_{t}^{(k)}\right)
$$

$c_{t}$ is the normalized factor such that $\sum_{k} \Pi_{t}(k)=1, \epsilon_{t}^{(k)}=$ $y_{t}-X^{(k)} \hat{h}_{t \mid t-1}$ is the innovations sequence from the Kalman filter tuned to $X_{t}=X^{(k)}$ and $\Omega_{t}^{(k)}=X^{(k)} \Sigma_{t \mid t-1} X^{(k)^{T}}+\sigma^{2} I$ 
is its associated covariance. Thus, the mean channel estimate and its associated covariance at time $t$ are

$$
\begin{aligned}
\hat{h}_{t \mid t} & =\sum_{k} \Pi_{t}(k) \hat{h}_{t \mid t}^{(k)}, \\
\Sigma_{t \mid t} & =\sum_{k} \Pi_{t}(k)\left\{\Sigma_{t \mid t}^{(k)}+\hat{h}_{t \mid t}^{(k)}\left(\hat{h}_{t \mid t}^{(k)}\right)^{T}\right\}-\hat{h}_{t \mid t} \hat{h}_{t \mid t}^{T} .
\end{aligned}
$$

In effect, we collapse the $K$ Kalman filters' estimates back to one each time. The estimated transmitted codeword at time $t$ is that which maximizes the APP.

From observation, we notice that most of the a posteriori probabilities that $X_{t}=X^{(k)}$ for $k=1, \ldots, K$ are very small. In fact, most of them are almost zero. Thus, the contribution of the channel estimates after being weighted by the small a posteriori probabilities to the mean channel estimate will be insignificant. Motivated by this observation, we will modify this receiver so that it will not compute the filtered channel estimate for the codeword with small APP. Thus, we can reduce the complexity of the receiver with little penalty on performance. The modified receiver will works as follow. After computing the APP that $X_{t}$ takes on a value in $\chi$ as in (30), $S$ codewords which have the greatest a posteriori probabilities will be selected, where $1 \leq S \leq K$. Lets $s$ denotes a member of the set of $S$ survivors. The a posteriori probabilities are then normalized so that $\sum_{s} \Pi_{t}(s)=1$. For each of these survivor paths, a filtered channel estimate, $\hat{h}_{t \mid t}^{(s)}$ and its associated covariance $\Sigma_{t \mid t}^{(s)}$ are calculated. These estimates are then combined to yield a single channel estimate and its covariance for time $t$. Thus

$$
\begin{aligned}
\hat{h}_{t \mid t} & =\sum_{s} \Pi_{t}(s) \hat{h}_{t \mid t}^{(s)} \\
\Sigma_{t \mid t} & =\sum_{s} \Pi_{t}(s)\left\{\Sigma_{t \mid t}^{(s)}+\hat{h}_{t \mid t}^{(s)}\left(\hat{h}_{t \mid t}^{(s)}\right)^{T}\right\}-\hat{h}_{t \mid t} \hat{h}_{t \mid t}^{T}
\end{aligned}
$$

This receiver has the flavor of the M-algorithm technique but it is not the same since at the end of each codeword period, this receiver retains only one channel estimate while the M-algorithm retains $S$ channel estimates. The complexity of this receiver is $O(S)$. When $S=K$ this receiver corresponds to the unmodified APP receiver as described previously.

2) Iterative EM Receiver: Another receiver which we proposed is the iterative receiver. In this approach, we use the expectation-maximization algorithm to iteratively maximize the $\log$-likelihood function, $\log p\left(y_{t} \mid y_{0}, \ldots, y_{t-1}, X_{t}=X^{(k)}\right)$. The iteration is per-codeword basis. When the channel is known, the log-likelihood is

$$
\begin{aligned}
L(t) & =\log p\left(y_{t} \mid h_{t}, X_{t}\right) \\
& =-\sigma^{-2}\left\|y_{t}-X_{t} h_{t}\right\|^{2}-M \log \sigma^{2} .
\end{aligned}
$$

Since the knowledge of the channel $h_{t}$ is not available at the receiver, the conditional expectation of this log-likelihood given the present and past measurements is used instead as the objective cost function

$$
\begin{aligned}
J_{t}\left(X_{t}, \hat{h}_{t \mid t}, \Sigma_{t \mid t}\right) & =E\left\{L(t) \mid y_{0}, \ldots, y_{t}\right\} \\
& =-\epsilon_{t}^{T} \Omega_{t}^{-1} \epsilon_{t}-\log \operatorname{det} \Omega_{t}
\end{aligned}
$$

where $\epsilon_{t}=y_{t}-X_{t} \hat{h}_{t \mid t}$ and $\Omega_{t}=X_{t} \Sigma_{t \mid t} X_{t}^{T}+\sigma^{2} I$. This motivates the iterative scheme whereby the conditional expectations is computed using $X_{t}=X^{\left(\hat{k}_{\ell}\right)}$ and then maximized $J_{t}$ over all codewords to yield $X^{\left(\hat{k}_{\ell+1}\right)}$. The iterative process for determining the transmitted codeword at time $t$ can be described in detail as follows.

Initialization: At the start of each iteration process, the channel estimate and its associated covariance are initialized to the Kalman filter one step prediction

$$
\begin{aligned}
& \tilde{h}_{t}^{(0)}=\hat{h}_{t \mid t-1}=A \hat{h}_{t-1 \mid t-1}+\mu \\
& \tilde{\Sigma}_{t}^{(0)}=\Sigma_{t \mid t-1}=A \Sigma_{t-1 \mid t-1} A^{T}+Q .
\end{aligned}
$$

The initial codeword is chose to maximize the cost function

$$
\hat{X}_{t}^{(0)}=\arg \max _{X_{t} \in \chi} J\left(X_{t}, \tilde{h}_{t}^{(0)}, \tilde{\Sigma}_{t}^{(0)}\right) .
$$

Iteration Update: We update from iteration $\ell-1$ to $\ell$ for $\ell \geq 1$ via

$$
\begin{aligned}
\tilde{h}_{t}^{(\ell)}= & \hat{h}_{t \mid t-1}+K_{t}^{(\ell)}\left(y_{t}-\hat{X}_{t}^{(\ell-1)} \hat{h}_{t \mid t-1}\right) \\
K_{t}^{(\ell)}= & \Sigma_{t \mid t-1}\left(\hat{X}_{t}^{(\ell-1)}\right)^{T} \\
& \cdot\left(\hat{X}_{t}^{(\ell-1)} \Sigma_{t \mid t-1}\left(\hat{X}_{t}^{(\ell-1)}\right)^{T}+\sigma^{2} I\right)^{-1} \\
\tilde{\Sigma}_{t}^{(\ell)}= & \Sigma_{t \mid t-1}-K_{t}^{(\ell)} \hat{X}_{t}^{(\ell-1)} \Sigma_{t \mid t-1} \\
\hat{X}_{t}^{(\ell)}= & \arg \max _{X \in \chi} J_{t}\left(X, \tilde{h}_{t}^{(\ell)}, \tilde{\Sigma}_{t}^{(\ell)}\right) .
\end{aligned}
$$

The algorithm terminates when the maximizing codeword is unaltered. We then set the values of $\hat{h}_{t \mid t}$ and $\Sigma_{t \mid t}$ to the final values of $\tilde{h}_{t}^{(\ell)}$ and $\tilde{\Sigma}_{t}^{(\ell)}$, respectively, and progress to codeword $t+1$. After the submission of this paper, we became aware of [16], which contains an iterative receiver based on Kalman filtering similar to ours. However, this receiver is designed specifically for decoding the Alamouti's space-time block code with two transmit and one receive antenna. Our proposed iterative EM receiver is more general.

\section{APPLICATION TO OFDM SySTEMS}

One important example of a diversity system is (OFDM) which is used in various wireless local area networks. In an OFDM system, data is transmitted over $L$ orthogonal frequency channels using a discrete Fourier transform (DFT). Within our context, we can regard the DFT operation as a type of "time-frequency" coding. Generally, a number of different data symbols (from a single user, or a number of different users) are transmitted simultaneously on subsets of the available channels. Here we will consider the single-user case, where one data symbol is transmitted on all $L$ channels simultaneously. This approach offers the maximal diversity gain. This is equivalent to using the precoder matrix $C$ equal to the $L$-vector of all $1 \mathrm{~s}$. Thus, corresponding to each data symbol (regarded as a member of a finite complex alphabet) $s_{t}$ we transmit a segment of $M=L$ time samples containing all $L$ carriers, and given by

$$
x_{t L+m}=\sum_{\ell=0}^{L-1} s_{t} e^{2 \pi i m \ell / L}
$$


for $m=0, \ldots, L-1$. In a standard OFDM system, a cyclic prefix is added to mitigate against intersymbol interference, and this is stripped off in the receiver. The recovered received samples then have the form

$$
r_{t L+m}=\sum_{\ell=0}^{L-1} s_{t} h_{t, \ell} e^{2 \pi i m \ell / L}+v_{t L+m}
$$

where $h_{t, \ell}$ denote the channel path gains as a function of symbol time $t$ and frequency channel $\ell$. Using this model, the frequency channels are spaced by an amount equal to the symbol rate. Here, $v_{\tau}$ is a zero-mean white Gaussian noise process. Now blocking the received samples into a vector of length $L$ corresponding to the symbol $s_{t}$, we have

$$
\tilde{y}_{t}=\tilde{X}_{t} \tilde{h}_{t}+\tilde{n}_{t}
$$

where $\tilde{X}_{t}$ is a $L \times L$ matrix with elements $\left[\tilde{X}_{t}\right]_{m, \ell}=$ $s_{t} e^{2 \pi i m \ell / L}$ and $\tilde{h}_{t}$ is an $L \times 1$ vector with elements $\left[\tilde{h}_{t}\right]_{\ell}=h_{t, \ell}$. We thus have placed this OFDM system model in the general diversity form (1). Note that the assumption that only one user symbol is transmitted over the $L$ channels is not restrictive as indicated in Section II-A.

\section{A. Physical Model for Delay-Doppler Spread Channels}

In this section, we relate our statistical channel gain model to a commonly used physical model for multipath propagation. This provides a parameterization of our state-space model in terms of specified physical parameters such as delay and doppler spreads for the channel. In [9], a physical model is used to characterize the correlation between the time-frequency gains $h_{t, \ell}$ in (40). This model is using the statistics of the channel and uses a block representation of the channel based on its autocorrelation matrix. For a classical Doppler power spectrum and exponential multipath intensity profile, the correlation between two symbols spaced in time and frequency, respectively, with $\Delta t$ and $\Delta f$ is given by [4]

$$
\phi(\Delta t, \Delta f)=\phi_{0} \phi_{t}(\Delta t) \phi_{f}(\Delta f)
$$

with $\phi_{0}>0$

$$
\phi_{t}(\Delta t)=J_{0}\left(\pi B_{d} \Delta t\right), \quad \phi_{f}(\Delta f)=\frac{1}{1+j 2 \pi T_{m} \Delta f} .
$$

Here, $J_{0}$ is the zeroth-order Bessel function of the first kind, with $B_{d}$ and $T_{m}$ being the Doppler and delay spreads, respectively, of the propagation channel. From (7), we can thus see that the $\tilde{P}$ matrix represents by itself the frequency correlation and $\tilde{A}^{t_{1}-t_{2}}$ is the time correlation between two symbols separated by a time shift equal to $\left(t_{1}-t_{2}\right)$. Thus immediately

$$
\tilde{P}_{k, l}=\frac{\phi_{0}}{1+j 2 \pi T_{m}(k-l) F_{s}}
$$

$F_{s}$ being the frequency spacing between two OFDM subcarriers.

Due to the separable nature of the physical channel correlation function (42), we take our state transition matrix $\tilde{A}$ to be of the form $\tilde{A}=a I$, where $a$ is a complex constant. Comparing the time correlation between two OFDM symbols separated by $k$ symbol periods, we need to have $a^{k}=J_{0}\left(\pi B_{d} k T_{s}\right)$ for every $k$, where $T_{s}$ is the symbol period. This is theoretically impossible, so an approximation has to be made for the $\left\{a_{k}\right\}_{k=0}^{D-1}$ to fit the $\left\{J_{0}\left(\pi B_{d} k T_{s}\right)\right\}_{k=0}^{D-1}, D$ being the number of OFDM symbols corresponding to the time length after which the correlation is considered to be insignificant, see [10]. This approximation is given by solving the least squares problem

$$
\min _{a} \sum_{k=0}^{D-1}\left|J_{0}\left(\pi B_{d} k T_{s}\right)-a^{k}\right|^{2} .
$$

This problem can be solved for various values of normalized Doppler spreads $\left(B_{d} T_{s}\right)$ and the corresponding values of $a$ used in the state-space model. One could also choose to include additional "modes" in $\tilde{A}$ to obtain a better approximation, but we do not provide details here.

As observed in [9], for some systems the effective amount of diversity can be less than $L$. This is manifest by the resulting covariance matrix $\tilde{P}$ not being strictly positive definite. In this case, we can reduce the dimension of the problem to $\tilde{L}$ the effective diversity of the channel, which is given by the number of nonzero eigenvalues of $\tilde{P}$. This is a standard subspace technique and is described in [9].

\section{Simulations}

We considered a single user OFDM system with 16 carriers. The data sequence is arranged into a block of 16 OFDM symbols. Each symbol is transmitted over the 16 orthogonal frequency channels using the DFT. The first symbol of each OFDM block will be used as pilot symbol to generate an estimate to initialize the Kalman filters. We assumed that the fading channels are independent from block to block. However, within each block, the time-frequency covariance of the fading channels between any two symbols is given by the model in Section IV. We assumed the channel is undergone Rayleigh fading, (i.e., $\mu=0$ ). In this simulation, we used $B_{d} T_{s}=0.25$. This Doppler spread value corresponds to a fast time-varying channel where the temporal correlation between any two consecutive symbols is only 0.852 . Even with such low temporal correlation in the channels, a significant performance gain can be attained by exploiting this time correlation as will be shown later in the simulation results. We examined two cases when $T_{m} F_{s}=0.5$ and $T_{m} F_{s}=0.025$. These values of $T_{m} F_{s}$ represent fading channels that are highly decorrelated and highly correlated in frequency dimension, respectively.

In this simulation, we compared the performance of the proposed techniques with the NCML, MAP, and GLRT receivers. In addition, we also compared them with the performance of the coherent ML receiver which has ideal channel state information (CSI). The performance of the coherent ML receiver will be used as the benchmark and we will see how close the performance of the proposed receivers can approach this bound. The PSP and the M-algorithm receivers perform sequence estimation on the whole OFDM block of 16 symbols while the APP and the iterative EM receivers perform symbol by symbol detection. The M-algorithm receiver with two survivors (i.e., $S=2$ ) and the unmodified APP receiver (i.e., the channel estimate is the weighted sum of the estimates from all the Kalman filters) are used in the simulation.

As from (41), the codeword $\tilde{X}_{t}$ has elements $\left[\tilde{X}_{t}\right]_{m, \ell}=$ $s_{t} e^{2 \pi i m \ell / L}$. In this simulation, we considered the case where the data symbol $s_{t} \in\{ \pm 1\}$. Thus, the codebook consists of 


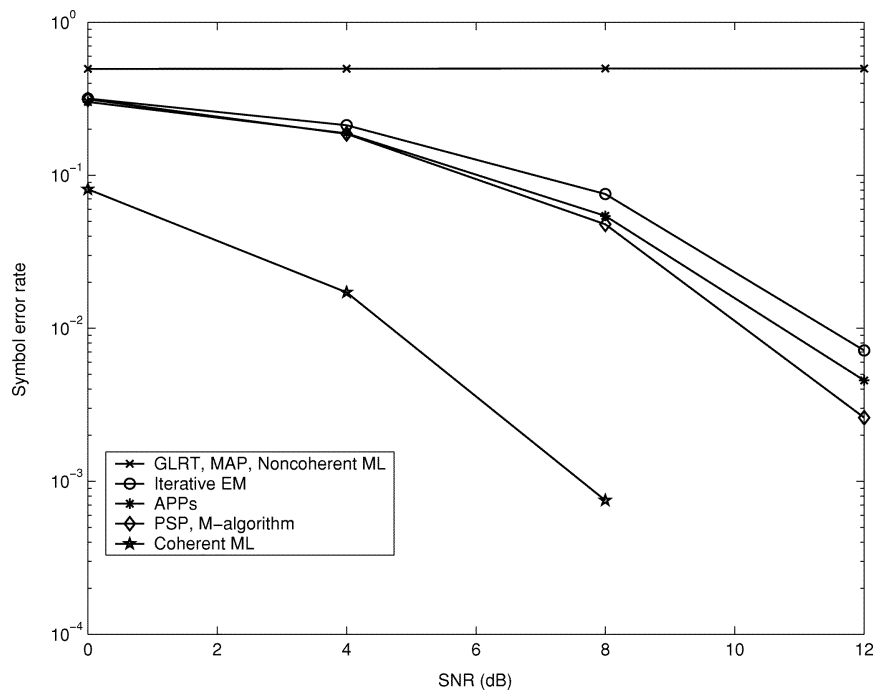

Fig. 4. Codes $= \pm F$ with $B_{d} T_{s}=0.25$ and $T_{m} F_{s}=0.5$.

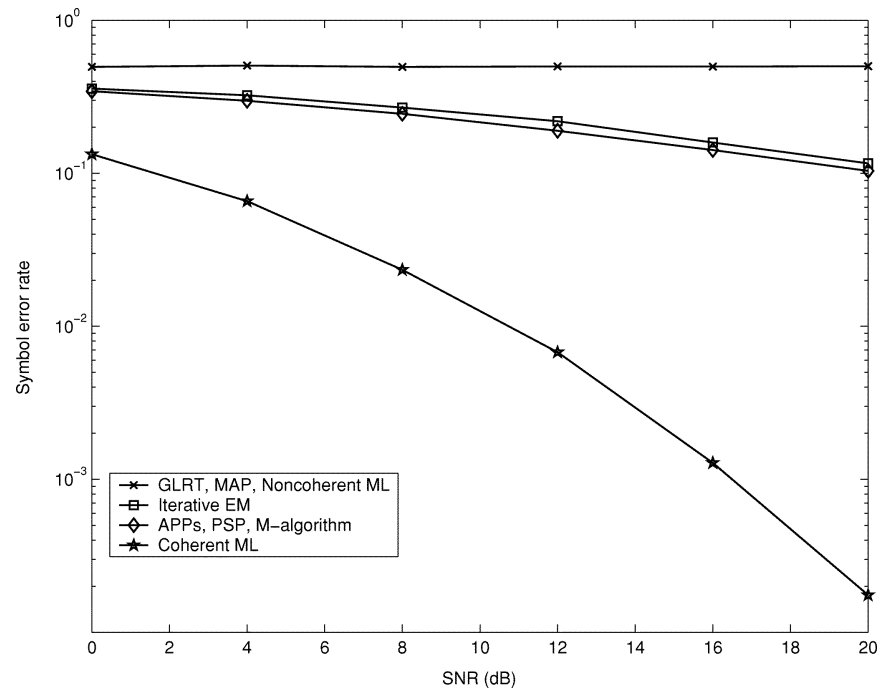

Fig. 5. Codes $= \pm F$ with $B_{d} T_{s}=0.25$ and $T_{m} F_{s}=0.025$.

two codes which are $\pm F$ where $F$ is the Fourier matrix. Fig. 4 shows the performance of the receivers for the fading channel with $B_{d} T_{s}=0.25$ and $T_{m} F_{s}=0.5$. The symbol-error rate (SER) is plotted for different values of signal-to-noise ratio (SNR), where the SNR is defined as the average received SNR per symbol. The standard GLRT, MAP, and NCML receivers all have an SER $=0.5$ for all SNs. This is because by using two codes which are scalar multiple of each other, the decision metrics of these receivers are indistinguishable (for the Rayleigh-fading channels) between the two codewords as discussed in [12] and [14]. Since the proposed receivers exploit the time correlation, then by using a pilot symbol to provide an estimate to initialize the Kalman filters, the proposed receivers do not suffer the same problem as those conventional noncoherent receivers. As shown in Fig. 4, at the SER $=10^{-2}$ the performance of the proposed receivers is within $5-7 \mathrm{~dB}$ of the coherent ML receiver. However, as the frequency correlation is increased, the performance of the proposed techniques is significantly reduced as shown in Fig. 5 for the highly

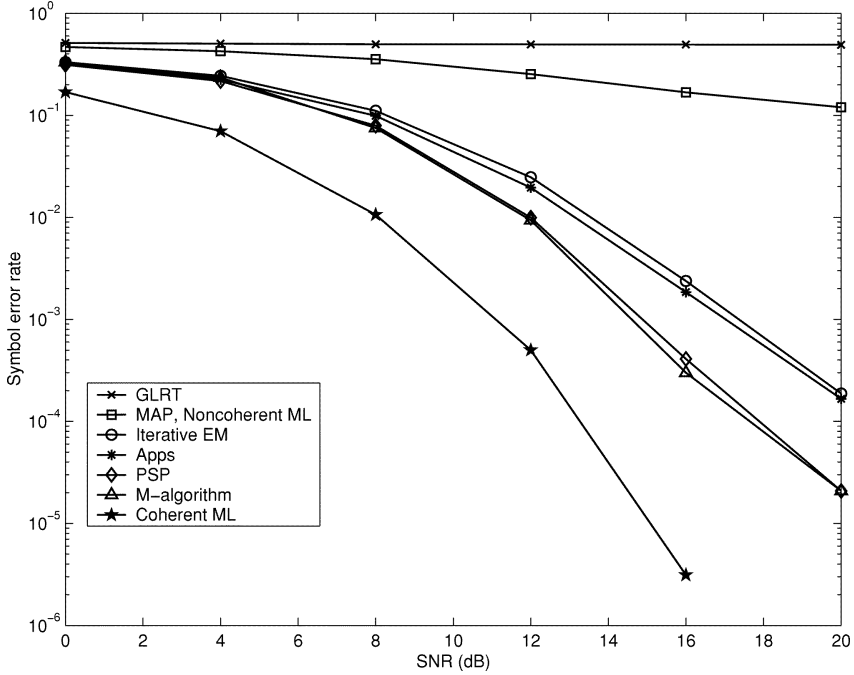

Fig. 6. Codes $=F$ and $U F$ with $B_{d} T_{s}=0.25$ and $T_{m} F_{s}=0.5$.

frequency correlated channel with $T_{m} F_{s}=0.025$. The reason for this huge degradation in performance is that as the channel become highly correlated in frequency, the diversity provided from the frequency domain is reduced. Thus, the probability that most of the channel coefficients faded at the same time is high, resulting in higher probability of error in detecting the transmitted code. Since the proposed receivers perform joint data detection and channel tracking, once an error is occurred it is likely to cause error propagation resulting in further error for the subsequence symbols in the block. The performance of the coherent ML receiver is also reduced in this case. However, this is mainly due to the loss in the diversity rather than the error propagation problem since it has ideal CSI. This suggests that using codes which are scalar multiples of each other is not optimal for noncoherent detection.

To prevent the above problem, we chose two codes which are not scalar multiple of each other. The two codes are $F$ and $U F$ matrices, where $U$ is an arbitrarily unitary matrix. Fig. 6 shows the performance of the receivers using the new codebook for the fading channel with $B_{d} T_{s}=0.25$ and $T_{m} F_{s}=0.5$. The GLRT receiver still has the SER $=0.5$ for this case since the orthogonal projector $P_{k}^{\perp}$ to the code $X^{(k)}$ is equal to zero for both codes. The performance of the MAP and the NCML receivers are still very poor. However, with this set of codes, the proposed receivers perform very well. At the SER $=10^{-3}$ the proposed techniques obtained a performance which is within $4 \mathrm{~dB}$ of the coherent ML receiver. Unlike the former set of codes, using these new codes reduces the performance gap between the proposed receivers and the coherent ML receiver when the fading channels are highly frequency correlated as shown in Fig. 7 for $T_{m} F_{s}=0.025$. We also note an interesting observation that the performance of the MAP and NCML is improving as the fading channels are more correlated. This can be explained by examining the decision metrics of these receivers.

The simulation results for all the scenarios considered above confirm the superiority of the proposed receivers to other standard noncoherent receivers. They demonstrate the usefulness of exploiting the temporal and diversity correlations in the channels at the receivers. Simulations above show that even in fast time-varying channels where the temporal correlation is low, 


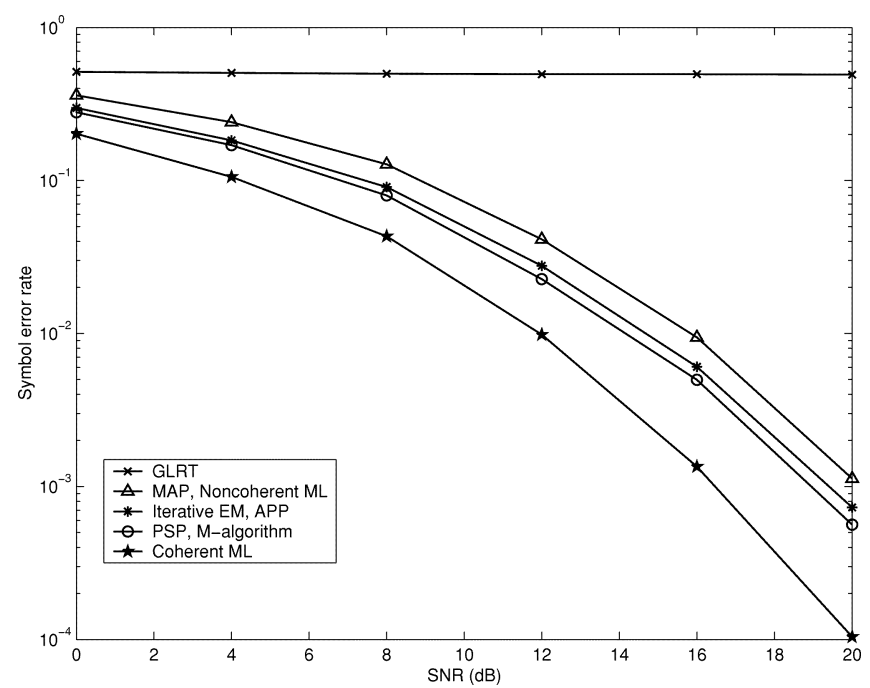

Fig. 7. Codes $=F$ and $U F$ with $B_{d} T_{s}=0.25$ and $T_{m} F_{s}=0.025$.

by exploiting such correlation could significant improve the receiver's performance. By utilizing the temporal correlation, the proposed receivers obtain a performance which is within few decibels of the coherent receiver.

The above observations are pertinent from the point of code design for incoherent systems with temporal channel correlations. It appears that an approach consisting of modulation followed by a fixed diversity code will perform poorly. Such an observation is supported by previous work such as [6] as an example which does not seal with such correlation. We believe there is a deeper issue at hand here when temporal correlation is included in the signal model, and we are currently investigating both performance characterization and code design in this case.

\section{CONCLUSION}

This paper has introduced a general state-space model for a general diversity communications system with time correlated flat fading. Examples of diversity systems which fall within this framework include space-time coded systems, OFDM systems, CDMA systems and hybrids of these systems. The model permits the design of a number of time-recursive noncoherent receivers based either on sequence estimation or on symbol by symbol estimation. The receivers considered include PSP, M-algorithm, two APP techniques, and a per-symbol iterative technique based on the EM algorithm. As an example of the utility of the approach, we examine an OFDM system model based on a physical delay doppler spread propagation medium, and we have shown how to approximate the resulting channel statistics by our model. The various receivers are compared with conventional designs which do not exploit the channel time correlations. These simulations have suggested that there can be significant gains in performance by incorporating time correlation into the signal model and the resulting receiver designs. Some implications for diversity code design in the noncoherent case are also briefly noted.

\section{ACKNOWLEDGMENT}

The authors would like to acknowledge Dr. J. Choi, who introduced us to the general space-time problem.

\section{REFERENCES}

[1] C. R. Johnson Jr. et al., "Blind equalization using the constant modulus criterion: A review," Proc. IEEE, vol. 86, no. 10, pp. 1927-1949, Oct. 1998.

[2] I. E. Telatar, "Capacity of multi-antenna Gaussian channels," AT\&T Bell Laboratories, Lucent Technologies, Murray Hill, NJ, 1995.

[3] G. J. Foschini and M. J. Gans, "On limits of wireless communications in a fading environment when using multiple antennas," Wireless Pers. Commun., vol. 6, no. 3, pp. 311-335, Mar. 1998.

[4] W. C. Jakes, Jr., Microwave Mobile Communications, W. C. Jakes Jr., Ed. New York: Wiley, 1974.

[5] N. Sellami, I. Fijalkow, and M. Siala, "Low-complexity iterative receiver for space-time coded signals over frequency selective channels," EURASIP J. Applied Signal Processing, Special Issue on Space-Time Coding and its Applications, no. 5, pp. 517-524, May 2002.

[6] M. Brehler and M. K. Varanasi, "Asymptotic error probability analysis of quadratic receivers in rayleigh fading channels with application to a unified analysis of coherent and noncoherent space-time receivers," IEEE Trans. Inform. Theory, vol. 47, pp. 2383-2399, Sept. 2001.

[7] A. Duel-Hallen, J. Holtzman, and Z. Zvonar, "Multiuser detection for CDMA systems," IEEE Pers. Commun., vol. 2, pp. 46-58, Apr. 1995.

[8] V. K. Nguyen and L. B. White, "Interference cancellation schemes for CDMA systems," in Proc. Information, Decision and Control 2002 Conf., Adelaide, Feb. 2002, p. 20.

[9] E. Jaffrot, M. Siala, and I. Fijalkow, "Maximum a posteriori semi-blind channel estimation for OFDM systems operating on highly frequency and time selective channels," IEEE Trans. Commun., submitted for publication.

[10] E. Jaffrot, V. K. Nguyen, M. Soamiadana, L. B. White, and I. Fijalkow, "Symbol by symbol reduced complexity highly selective OFDM channel estimation," in Proc. EUSIPCO 2002, Toulouse, France, Sept. 2002.

[11] V. Tarokh, N. Seshadri, and A. R. Calderbank, "Space-time codes for high data rate wireless communication: Performance criterion and code construction," IEEE Trans. Inform. Theory, vol. 44, pp. 744-765, Mar. 1998.

[12] B. M. Hochwald, T. L. Marzetta, T. J. Richardson, W. Sweldens, and R. Urbanke, "Systematic design of unitary space-time constellations," IEEE Trans. Inform. Theory, vol. 46, pp. 1962-1973, Sept. 2000.

[13] D. Agrawal, T. J. Richardson, and R. Urbanke, "Multiple-antenna signal constellations for fading channels," IEEE Trans. Inform. Theory, vol. 47, pp. 2618-2626, Sept. 2001

[14] D. Warrier and U. Madhow, "Spectrally efficient noncoherent communication," IEEE Trans. Inform. Theory, vol. 48, pp. 651-668, Mar. 2002.

[15] E. Visotsky and U. Madhow, "Noncoherent multiuser detection for CDMA systems with nonlinear modulation: A non-Bayesian approach," IEEE Trans. Inform. Theory, vol. 47, pp. 1352-1367, May 2001.

[16] Z. Liu, X. Ma, and G. B. Giannakis, "Space-time coding and Kalman filtering for time-selective fading channels," IEEE Trans. Commun., vol. 50, pp. 183-186, Feb. 2002.

[17] S. M. Kay, Fundamentals of Statistical Signal Processing: Estimation Theory. Englewood Cliffs, NJ: Prentice-Hall, 1993.

[18] R. Raheli, A. Polydoros, and C. Tzou, "Per-survivor processing: A general approach to MLSE in uncertain environments," IEEE Trans. Commun., vol. 43, pp. 354-364, Feb./Mar./Apr. 1995.

[19] T. Hashimoto, "A list-type reduced-constraint generalization of the viterbi algorithm," IEEE Trans. Inform. Theory, vol. IT-33, pp. 866-876, Nov. 1987.

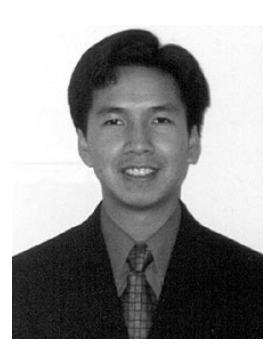

Van Khanh Nguyen was born in Vietnam in 1978. $\mathrm{He}$ received the B.E. degree (honors) in computer system engineering in 2000 and is currently working toward the Ph.D. degree in the Department of Electrical and Electronic Engineering, University of Adelaide, Adelaide, Australia.

$\mathrm{He}$ is currently with the Centre for Internet Technology Research, University of Adelaide. His research interests include multiuser detection, space-time coding, joint decoding and channel estimation, broadband wireless communication systems, multicarrier transmission, and adaptive modulation. 


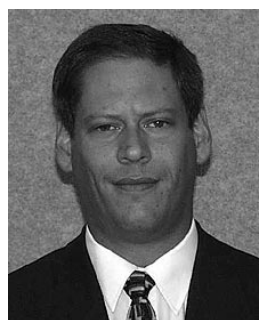

Langford B. White (S'83-M'88-SM'00) received the B.S. degree in mathematics, the B.E. (honors) and $\mathrm{Ph} . \mathrm{D}$. degrees in electrical engineering from the University of Queensland, Brisbane, Australia, in 1984, 1985 , and 1989 respectively.

From 1986 to 1999, he worked for the Defence Science and Technology Organization, Salisbury, South Australia, in the areas of radar and communications electronic warfare. Since 1999, he has been a Professor of communication networks in the Department of Electrical and Electronic Engineering, University of Adelaide, Adelaide, Australia. His current research interests include communications signal processing, wireless data networks, and the internet.

Prof. White was awarded the Australian Telecommunications and Electronics Research Board Medal for Outstanding Young Investigator in 1994.

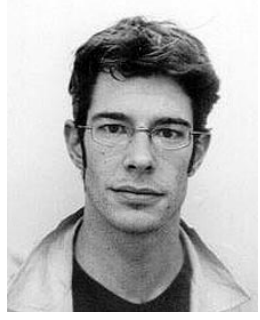

Emmanuel Jaffrot was born in Nantes, France, in 1973. He received B.E. and M.S. degrees in signal processing for digital communications from the Ecole Nationale Supérieure de l'Electronique et de ses Applications and Université de Cergy Pontoise, Cergy, France, in 1996 and the Ph.D. degree from Ecole Nationale Supérieure des Télécommunications (ENST), Paris, France, in 2000.

Since 2001, he has been a Member of the UEI Laboratory (ENSTA, Paris, France) and an Assistant Professor. His research interests are currently about multicarrier systems, ultrawideband systems, channel estimation, and smart antennas.

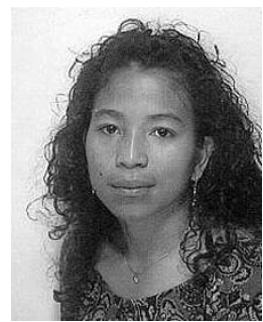

Marcella Soamiadana was born in Madagascar in 1974. She received the TIS (ENSEA-UCP) Master degree from Ecole Nationale Supérieure de l'Electronique et ses Applications (ENSEA) and Université de Cergy Pontoise, Cergy, France, in 2001 and is currently working toward the Ph.D. degree in the ETIS Laboratory ENSEA-UCP and ENSTA advised by I. Fijalkow and E. Jaffrot in "Multisensor receivers for OFDM System"

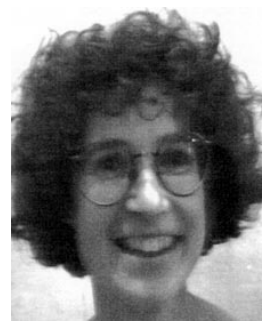

Inbar Fijalkow (M'96) received the B.E. and Ph.D. degrees from Ecole Nationale Supérieure des Télécommunications (ENST), Paris, France, in 1990 and 1993, respectively.

Since 1994, she has been a Member of Laboratory ETIS/ENSEA, University of Cergy Pontoise-CNRS, France, as a Professor. Her current research interests are in signal processing for digital communications: adaptive and iterative (turbo) processing, channel estimation, and equalization for link adaptation.

Dr. Fijalkow is a Member of the Board of the GDR ISIS, the CNRS Research Group on signal image and vision processing. She has been an Associate Editor of the IEEE TRANSACTIONS ON Signal PROCESSING (2000-2003). 